Yu. L. Kalinovsky, V. D. Toneev, A. V. Friesen

\title{
Phase diagram of baryon matter in the SU(2) Nambu - Jona-Lasinio model with a Polyakov loop
}

Uspekhi Fizicheskikh Nauk, 2016, 186:4, 387-403

\section{References}

[1] Greiner W., Schäfer A., Quantum Chromodynamics, Springer-Verlag, Berlin, 1994

[2] Ejiri S., Nucl. Phys. B Proc. Suppl., 94 (2001), 19 dol WEB of SCIENCETM

[3] arXiv: 1111.1710 dol WEB OF SCIENCETM

[4] Wu L.-K., Luo X.-Q., Chen H.-S., Phys. Rev. D, 76 (2007), 034505 doil WEB OF SCIENCETM

[5] Nambu Y., Jona-Lasinio G., Phys. Rev., 122 (1961), 345 dol

[6] Nambu Y., Jona-Lasinio G., Phys. Rev., 124 (1961), 246 doi

[7] Gell-Mann M., Lévy M., Nuovo Cimento, 16 (1960), 705 doi]

[8] Eguchi T., Phys. Rev. D, 14 (1976), 2755 doi

[9] Kikkawa K., Prog. Theor. Phys., 56 (1976), 947 dol]

[10] Volkov M. K., Ebert D., Sov. J. Nucl. Phys., 36 (1982), 736

[11] Ebert D., Volkov M. K., Z. Phyz. C, 16 (1983), 205 dol WEB OF SCIENCE

[12] Volkov M. K., Ann. Physics, 157 (1984), 282 dol WEB OF SCIENCET

[13] Volkov M. K., Sov. J. Part. Nucl., 17 (1986), 186

[14] Hatsuda T., Kunihiro T., Phys. Rev. Lett., 55 (1985), 158 dol WEB OF SCIENCEXM

[15] Hatsuda T., Kunihiro T., Phys. Lett. B, 185 (1987), 304 dol WEB OF SCIENCE

[16] Kunihiro T., Phys. Lett. B, 219 (1989), 363 dol WEB of SCIENCETN

[17] Klevansky S. P., Rev. Mod. Phys., 64 (1992), 649 dol WEB OF SCIENCEMW

[18] Bernard V., Meissner U.-G., Zahed I., Phys. Rev. D, 36 (1987), 819 dei WEB OF SCIENCE

[19] Bernard V., Meissner U.-G., Zahed I., Phys. Rev. Lett., 59 (1987), 966 dol WEB OF SCIENCETX

[20] Christov C. V., Ruiz-Arriola E., Goeke K., Acta Phys. Polon. B, 22 (1991), 187 WEB OF SCIENCETM

[21] Hüfner J., Klevansky S. P., Zhuang P., Acta Phys. Polon. B, 25 (1994), 85 WEB OF SCIENCETW

[22] Kalinovsky Yu. L., Friesen A. V., Phys. Part. Nucl. Lett., 12 (2015), 737 do?

[23] Efimov G. V., Ivanov M. A., Sov. J. Part. Nucl., 20 (1989), 479

[24] Andrianov A. A., Espriu D., Tarrach R., Nucl. Phys. B, 533 (1998), 429 WEB OF SCIENCETM

[25] 60 (1999), 039901, Erratum deil doi WEB OF SCIENCEET

[26] Dyakonov D. I., Petrov V. Yu., Nucl. Phys. B, 272 (1986), 457 doil Web of SCIENCEM

[27] Buballa M., Krewald S., Phys. Lett. B, 294 (1992), 19 dol WEB OF SCIENCEW

[28] Anikin I. V., Dorokhov A. E., Tomio L., Phys. Part. Nucl., 31 (2000), 509

[29] Polyakov A. M., Phys. Lett. B, 72 (1978), 477 dol WEB OF SCIENCENW

[30] Meisinger P. N., Ogilvie M. C., Phys. Lett. B, 379 (1996), 163 del Web of SCIENCETM

[31] Meisinger P. N., Miller T. R., Ogilvie M. C., Phys. Rev. D, 65 (2002), 034009 dof WEB OF SCIENCE

[32] Mócsy A., Sannino F., Tuominen K., Phys. Rev. Lett., 92 (2004), 182302 do? WEB OF SCIENCETM

[33] Fukushima K., Phys. Lett. B, 591 (2004), 277 dol WEB OF SCIENCENM

[34] Ratti C., Thaler M. A., Weise W., Phys. Rev. D, 73 (2006), 014019 dol WEB OF SCIENCENW

[35] Hansen H. et al., Phys. Rev. D, 75 (2007), 065004 dol WEB OF SCIENCETM

[36] Rößner S., Ratti C., Weise W., Phys. Rev. D, 75 (2007), 034007 dol Web of SCIENCENW

[37] Costa P. et al., Phys. Rev. D, 81 (2010), 016007 dol WEB OF SCIENCEM 
[38] Fukushima K., Phys. Rev. D, 77 (2008), 114028 doi WEB OF SCIENCEMT

[39] Lugones G. et al., Phys. Rev. D, 81 (2010), 085012 dol WEB OF SCIENCETM

[40] Lenzi C. H. et al., Phys. Rev. C, 82 (2010), 015809 dol WEB OF SCIENCEM

[41] Dexheimer V. et al., Phys. Rev. C, 87 (2013), 015804 dol WEB OF SCIENCEN

[42] Borsányi S. et al., JHEP, 2010, no. 09, 073 del WEB OF SCIENCEM

[43] de Forcrand P., Philipsen O., Nucl. Phys. B, 642 (2002), 290 dol WEB OF SCIENCETM

[44] D'Elia M., Lombardo M.-P., Phys. Rev. D, 67 (2003), 014505 doi WEB of SCIENCETM

[45] D'Elia M., Di Renzo F., Lombardo M.-P., Phys. Rev. D, 76 (2007), 114509 do? WEB OF SCIENCEN

[46] Kogut J. B., Sinclair D. K., Phys. Rev. D, 70 (2004), 094501 dol Web of ScienceTy

[47] Cea P. et al., Phys. Rev. D, 80 (2009), 034501 dol WEB OF SCIENCETM

[48] Sakai Y. et al., Phys. Rev. D, 82 (2010), 076003 dol WEB OF SCIENCETM

[49] arXiv: 1104.2394 dol WEB OF SCIENCE'T

[50] Ruivo M. C., Costa P., de Sousa C. A., Phys. Rev. D, 86 (2012), 116007 do] WEB OF SCIENCE TW

[51] Sugano J. et al., Phys. Rev. D, 90 (2014), 037901 dol WEB OF SCIENCETI

[52] Friesen A. V., Kalinovsky Yu. L., Toneev V. D., Int. J. Mod. Phys. A, 30 (2015), 1550089 doI WEB OF SCIENCENM

[53] Vogl U., Weise W., Prog. Part. Nucl. Phys., 27 (1991), 195 doi Web of SCIENCETM

[54] Buballa M., Phys. Rep., 407 (2005), 205 del WEB OF SCIENCENM

[55] Lourenco O., Phys. Rev. D, 85 (2012), 097504 dol] WEB OF SCIENCETM

[56] Dutra M. et al., Phys. Rev. D, 88 (2013), 114013 doil WEB OF SCIENCETM

[57] Fukushima K., Phys. Rev. D, 78 (2008), 114019 dei WEB OF SCIENCENW

[58] Sakai Y. et al., Phys. Rev. D, 78 (2008), 076007 dol WEB OF SCIENCENM

[59] Carignano S., Nickel D., Buballa M., Phys. Rev. D, 82 (2010), 054009 doil WEB OF SCIENCETW

[60] Steinheimer J., Schramm S., Phys. Lett. B, 696 (2011), 257 doil WEB OF SCIENCETM

[61] Phys. Part. Nucl. Lett., 11 (2014), 342 dol

[62] Phys. Part. Nucl. Lett., 10 (2013), 660 dol

[63] Simonov Yu. A., Phys. Usp., 39 (1996), 313 Math-Net.Ru doil do?

[64] arXiv: hep-ph/0203271

[65] Pisarski R. D., Phys. Rev. D, 62 (2000), 111501(R) dol WEB OF SCIENCETM

[66] Boyd G. et al., Nucl. Phys. B, 469 (1996), 419 dof WEB OF SCIENCEM

[67] Rößner S. et al., Nucl. Phys. A, 814 (2008), 118 dol WEB OF SCIENCENY

[68] Dexheimer V. A., Schramm S., Nucl. Phys. A, 827 (2009), 579c del WEB OF SCIENCEM

[69] Dexheimer V. A., Schramm S., Phys. Rev. C, 81 (2010), 045201 dol WEB of SCIENCE

[70] Panero M., Phys. Rev. Lett., 103 (2009), 232001 dol WEB OF SCIENCETM

[71] Meisinger P. N., Ogilvie M. C., Miller T. R., Phys. Lett. B, 585 (2004), 149 do] WEB OF SCIENCE

[72] Karsch F., Laermann E., Peikert A., Phys. Lett. B, 478 (2000), 447 doi WEB OF SCIENCENX

[73] Karsch F., Nucl. Phys. A, 698 (2002), 199 dol

[74] arXiv: hep-ph/0701091 doi WEB OF SCIENCEN

[75] Kondo K.-I., Phys. Rev. D, 82 (2010), 065024 dol WEB OF SCIENCETW

[76] Roberge A., Weiss N., Nucl. Phys. B, 275 (1986), 734 dol WEB OF SCIENCENM

[77] Dutra M. et al., Phys. Rev. D, 88 (2013), 114013 do] WEB OF SCIENCENM

[78] Sugano J. et al., Phys. Rev. D, 90 (2014), 037901 dol WEB OF SCIENCEW

[79] Endrödi G. et al., JHEP, 2011:4 (2011), 1 del WEB OF SCIENCETX

[80] Kaczmarek O. et al., Phys. Rev. D, 83 (2011), 014504 do] WEB OF SCIENCETM

[81] arXiv: 1410.5758 dol WEB OF SCIENCE'T 
[82] Braguta V. V. et al., JETP Lett., 101 (2015), 732 Math-Net.Ru dol WEB OF SCIENCETM

[83] Cea P., Cosmai L., Papa A., Phys. Rev. D, 89 (2014), 074512 dol Web OF SCIENCETI

[84] Masayuki A., Koichi Y., Nucl. Phys. A, 504 (1989), 668 doid Web of science

[85] arXiv: nucl-th/0007030 dol WEB OF SCIENCEW

[86] Ejiri S. et al., Theor. Phys. Suppl., 153 (2003), 118 do?

[87] Gavai R. V., Gupta S., Phys. Rev. D, 71 (2005), 114014 del Web of SCIENCET⿱卄一

[88] Fodor Z., Katz S. D., JHEP, 2002, no. 03, 014 dol WEB OF SCIENCEW

[89] Fodor Z., Katz S. D., JHEP, 2004, no. 04, 050 do?

[90] Ali Khan A. et al. (CP-PACS Collab.), Phys. Rev. D, 64 (2001), 074510 do?

[91] Friesen A. V., Kalinovsky Yu. L., Toneev V. D., Int. J. Mod. Phys. A, 27 (2012), 1250013 dol WEB OF SCIENCEN

[92] Uhlenbeck G. E., Beth E., Physica, 3 (1936), 729 do?

[93] Beth E., Uhlenbeck G. E., Physica, 4 (1937), 915 do]

[94] Abuki H., Nucl. Phys. A, 791 (2007), 117 doi WEB OF SCIENCETM

[95] Hufner J. et al., Ann. Physics, 234 (1994), 225 doi WEB OF SCIENCETW

[96] Kapusta J. I., Finite-Temperature Field Theory, Cambridge Univ. Press, Cambridge, 1989

[97] Sakai Y. et al., Phys. Rev. D, 77 (2008), 051901 dol WEB OF SCIENCE

[98] Sakai Y. et al., Phys. Rev. D, 78 (2008), 036001 dol WEB OF SCIENCETM

[99] arXiv: 1105.1739 dol WEB OF SCIENCEMU

[100] Kouno H. et al., Phys. Rev. D, 83 (2011), 076009 doi WEB OF SCIENCETM

[101] Kashiwa K. et al., Phys. Rev. D, 79 (2009), 076008 dol WEB OF SCIENCETM

[102] arXiv: 1111.3839

[103] arXiv: 1111.3446

[104] Hell T. et al., Phys. Rev. D, 79 (2009), 014022 dol WEB OF SCIENCE'M

[105] Chatterjee B., Mishra H., Mishra A., Phys. Rev. D, 91 (2015), 034031 dol WEB OF SCIENCETX

[106] Fukushima K., Ruggieri M., Gatto R., Phys. Rev. D, 81 (2010), 114031 do? WEB OF SCIENCETW

[107] Braguta V. V. et al., JHEP, 2015:6 (2015), 094 do] WEB OF SCIENCETM

[108] Rehberg P., Klevansky S. P., Hüfner J., Phys. Rev. C, 53 (1996), 410 dod WEB OF SCIENCENW

[109] Friesen A. V., Kalinovsky Yu. L., Toneev V. D., Nucl. Phys. A, 923 (2014), 1 do? WEB OFSCIENCEN

[110] Phys. Part. Nucl. Lett., 9 (2012), 1 do?

[111] arXiv: nucl-th/0008041 doi WEB OF SCIENCE'M

[112] Fu W., Liu Y., Phys. Rev. D, 79 (2009), 074011 del Web of SCIENCE 